\title{
Occlusal analysis and management of a patient with vertigo: a case report
}

\author{
Kengo Torii* \\ Department of General Dentistry, School of Life Dentistry, Nippon Dental University, 1-9-20 Fujimi, Chiyoda-ku, Tokyo, 102-8159, Japan
}

\begin{abstract}
Although the relationship between aural symptoms and dental occlusion has been reported, the mechanism of vertigo still remains unclear. Occlusal improvement was performed on a patient with vertigo. Occlusal analysis was performed on the patient by mounting dental models on an articulator and identifying a deviation of the habitual occlusal position (HOP) from the biteplate-induced occlusal position (BPOP). The deviation was a semi-rotatory shift of 2.0 mm backward on the left side and $2.0 \mathrm{~mm}$ forward on the right side from BPOP. Occlusal modifications of the upper complete denture were performed to make HOP coincide with BPOP. The vertigo has not recurred till date after the 3-month follow-up period. The vertigo was thought to be caused by nystagmus along with the repeated semi-rotatory shift to HOP from BPOP.
\end{abstract}

\section{Introduction}

The relationships between aural and other symptoms and temporomandibular disorders (TMDs) have been reported [1-6]. In these reports, there were some descriptions regarding the relationship between dental occlusion and vertigo [1,2]. However, the mechanism of vertigo remains unclear. The present case might be useful to understand one of the mechanisms of vertigo.

\section{Case presentation}

A 75-year-old man presented with a chief complaint of looseness of his upper complete denture. He reported that he suddenly felt a strong impact on his left ear and developed such a severe rotatory vertigo 2 years ago that he had to be carried to the emergency department of a hospital. However, no abnormalities were found in his brain. $\mathrm{He}$ then visited an ENT clinic. After various tests, no abnormalities were found in his ears. He reported that he has been experiencing vertigo since that time, especially when he turns to the left, and he developed severe tinnitus in his left ear. Furthermore, he reported that while he was sitting on a stool and looking at a point on the wall in front of him, his visual point repeatedly moved from right to left. He also complained about a headache on the left side, pain behind the right eyeball, and a spasm on the right side of his face and right leg. The patient's medical history was unremarkable, except for high blood pressure. No impairment of mouth opening, deviation of the opening path, and no noises in either of the temporomandibular joints (TMJs) were detected. The patient reported tenderness in the right lateral pterygoid muscle, left posterior part of temporalis, and left occipitalis. The patient wore an upper complete denture, and with nine natural lower teeth, he wore a lower removable partial denture (Figures 1 and 2). For diagnosis, an anterior bite plate was fabricated using clear selfcuring acrylic resin (GC, Tokyo, Japan) (Figures 3 and 4). A habitual occlusal position (HOP) record was obtained by voluntary jaw closing using a vinylpolysiloxane bite registration material (GC, Tokyo, Japan). A bite plate-induced occlusal position (BPOP) record was obtained during voluntary jaw closing after wearing the bite plate for $5 \mathrm{~min}$ using the same material as in HOP. TMJ computed tomography images were taken using these records (Figure 5). No obvious differences in either position were noticed. To examine the difference between HOP and BPOP, two-dimensional measurements were performed on a modified articulator using previous records. The position of the recording disc was approximately the same as that of the condyle of TMJ. Therefore, the shift was caused by as one of the condyles. Semi-rotatory shifts from BPOP to HOP were recorded on the discs, with $2.0 \mathrm{~mm}$ of the shift on the left side and $2.0 \mathrm{~mm}$ on the right side, respectively (Figures 6 and 7). Following the clinical examination and the mandibular position analysis, the patient was diagnosed with myogenous type of TMD $[7,8]$. Because the occlusion in BPOP was unstable due to premature contact and lack of bilateral posterior occlusal contacts (Figure 8), the upper denture was modified by adding some self-curing acrylic resin to the occlusal surfaces of the posterior teeth on both sides (Figure 9). The patient was evaluated after 1 week. The vertigo did not develop, and

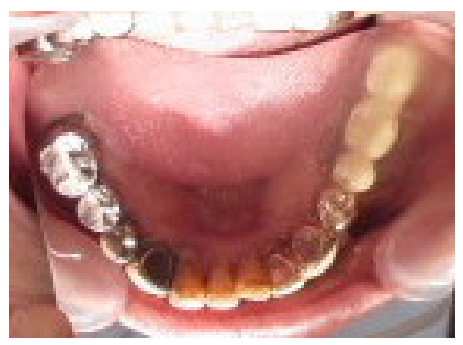

Figure 1. Lower dental arch.

Correspondence to: Kengo Torii, Department of General Dentistry, School of Life Dentistry, Nippon Dental University, 1-9-20 Fujimi, Chiyoda-ku, Tokyo, 102-8159, Japan, Tel: 054247 2966; E-mail: wbs89508@mail.wbs.ne.jp

Key words: vertigo, nystagmus, habitual occlusal position, bite plate-induced occlusal position

Received: November 20, 2016; Accepted: December 14, 2016; Published: December 16, 2016 


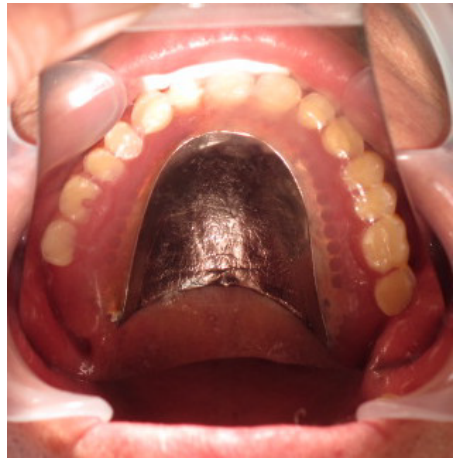

Figure 2. Upper complete denture.

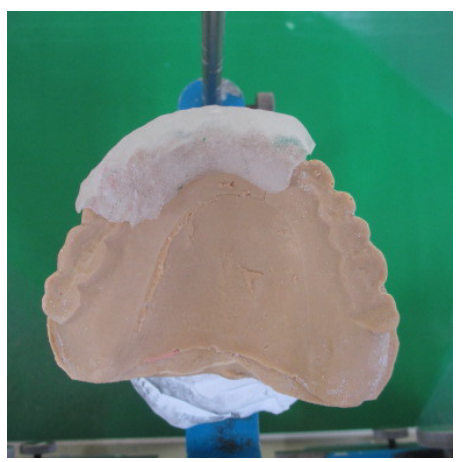

Figure 3. Anterior bite plate.

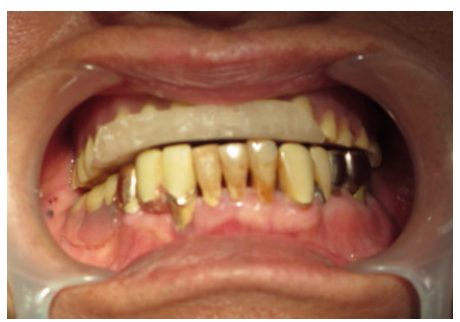

Figure 4. Wearing the bite plate.

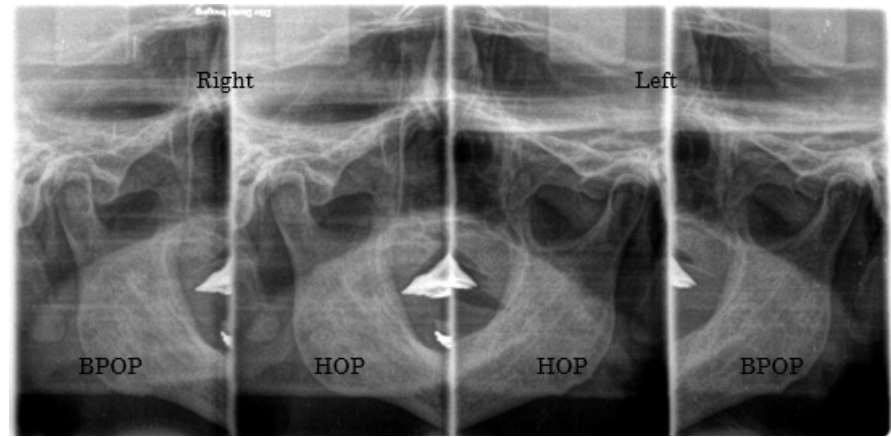

Figure 5. Bilateral tomographic image of TMJ in HOP and BPOP.

the tinnitus had diminished. The headache, spasm on the right side of the face, pain behind the right eye, and spasm of the right leg had all disappeared, with no recurrence till date after a 3-month follow-up period.

\section{Discussion}

Regarding vertigo, Myrhaug described that in vestibular type of vertigo, the violent dizzy attacks generally start with strong and intense tinnitus and are often associated with headaches, and such attacks can

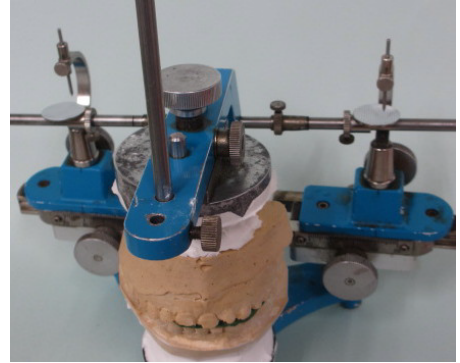

Figure 6. Mandibular position analyzer.

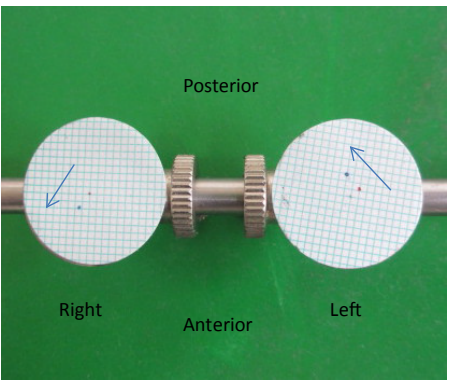

Figure 7. Recorded HOP and BPOP. The arrow indicates the shift from BPOP to HOP.

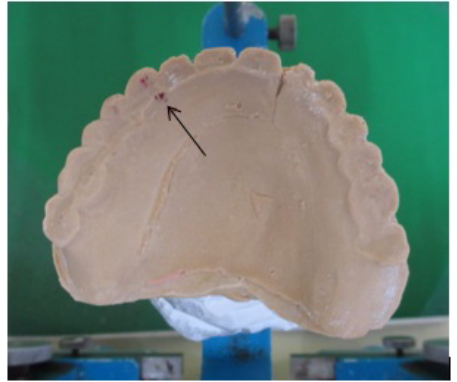

Figure 8. The arrow indicates the premature occlusal contact in BPOP.

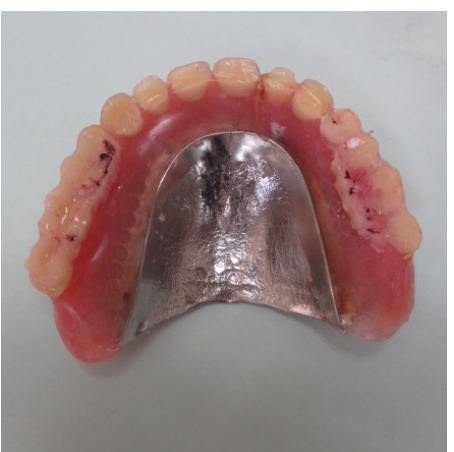

Figure 9. Modified bilateral posterior occlusal surfaces.

arise from sudden and severe movements of the footplate of the stapes. A ripple of the waves arise in the lymph of the inner ear, which gives rise to these reactions and exerts an intermittent pressure on the wall of the labyrinth. This causes vertigo [1]. However, it remains unclear how the intermittent pressure can cause vertigo. In the present case, the semi-rotatory shift, which is frequent, short, and cyclically repeated in mastication and other oral functions, would be recognized as the rotatory movement and would cause nystagmus, thereby giving the patient had an illusion of rotation and resulting in vertigo. Because the direction of nystagmus is the same as that of the rotating movement, and in the present case, the direction of the rotatory movement was to the left, the patient developed vertigo when he turned left. Torii and 
Chiwata reported a case of vertigo with a condylar shift of $3 \mathrm{~mm}$ on the right side and $1 \mathrm{~mm}$ on the left side, respectively [3]. That case may also have resulted from this aforementioned mechanism. On the other hand, the severe tinnitus in the left ear may be caused by the spasmodic synkinesis of the left tensor tympani and the left stapedial muscle with muscle spasm of the left temporalis because a stronger muscle force is needed to pull the mandible backward than it is to pull it forward [6]. The spasm of the right leg in the present case may be caused by compensating the imbalanced mandibular posture to maintain the body posture [5]. The present case suggests that the occlusal discrepancy between HOP and BPOP can affect various body functions.

\section{Acknowledgement}

Written consent was obtained from the patient for publication of the study.

\section{Competing and conflicting interests}

The author declares no competing interests.

\section{References}

1. Myrhaug H (1964) The incidence of ear symptoms in cases of malocclusion and temporo-mandibular joint disturbances. Br J Oral Surg 2: 28-32. [Crossref]

2. Rubinstein B (1993) Tinnitus and craniomandibular disorders-is there a link? Swed Dent J Suppl 95: 1-46. [Crossref]

3. Torii K, Chiwata I (2007) Occlusal management for a patient with aural symptoms of unknown etiology: a case report. J Med Case Rep 1: 85. [Crossref]

4. Stack B, Sims A (2009) The relationship between posture and equilibrium and the auriculotemporal nerve in patients with disturbed gait and balance. Cranio 27: 248260. [Crossref]

5. Torii K (2016) Coxalgia and temporomandibular disorders: a case report. Int Arch Med 9: 294 .

6. Torii K (2011) Tinnitus and temporomandibular disorders, In: Up to Date on Tinnitus, Fayez B Jr ed. Rijeka: In Tech :15-32.

7. Torii K, Chiwata I (2005) Relationship between habitual occlusal position and flat bite plane-induced occlusal position in volunteers with and without temporomandibular joint sounds. Cranio 23: 16-21.

8. Torii K, Chiwata I (2010) Occlusal adjustment using the bite plate-induced occlusal position as a reference position for temporomandibular disorders: a pilot study. Head \& Face Medicine 6: 5 .

Copyright: $(02016$ Torii K. This is an open-access article distributed under the terms of the Creative Commons Attribution License, which permits unrestricted use, distribution, and reproduction in any medium, provided the original author and source are credited. 